\title{
Intraburst and Interburst Signaling by Climbing Fibers
}

\author{
Jun Maruta, Robert A. Hensbroek, and John I. Simpson \\ Department of Physiology and Neuroscience, New York University School of Medicine, New York, New York 10016
}

\begin{abstract}
Although cerebellar Purkinje cell complex spikes occur at low frequency $(\sim 1 / \mathrm{s})$, each complex spike is often associated with a highfrequency burst $(\sim 500 / \mathrm{s})$ of climbing fiber spikes. We examined the possibility that signals are present within the climbing fiber bursts. By intracellularly recording from depolarized, nonspiking Purkinje cells in anesthetized pigmented rabbits, climbing fiber burst patterns were investigated by determining the number of components in the induced compound EPSPs during spontaneous activity and during visual stimulation. For our sample of 43 cells, $>70 \%$ of all EPSPs were of the compound type composed of two or three EPSPs. During spontaneous activity, the number of components in each compound EPSP was not related to the latency to the succeeding compound EPSP. Conversely, the number of components in each compound EPSP was related to its latency after the preceding compound EPSP. This latency increased from $0.62 \mathrm{~s}$ for one-component EPSPs to $1.69 \mathrm{~s}$ for compound EPSPs with four or more components. The effect of visual stimulation on the climbing fiber activity was studied in 19 floccular Purkinje cells whose low-frequency interburst climbing fiber response was modulated by movement about the vertical axis. During sinusoidal oscillation $\left(0.1 \mathrm{~Hz}, \pm 10^{\circ}\right)$, compound EPSPs with a larger number of components tended to be more prevalent during movement in the excitatory direction than in the inhibitory direction. Thus, climbing fibers can, in addition to modulation of their low interburst frequency, transmit signals in the form of the number of spikes within each high-frequency burst.
\end{abstract}

Key words: inferior olive; cerebellum; afterdepolarization; model; Purkinje neurons; complex spike

\section{Introduction}

Although cerebellar Purkinje cell complex spikes occur at low frequency $(\sim 1 / \mathrm{s})$, each complex spike is often associated with a high-frequency burst of climbing fiber spikes (Eccles et al., 1966; Armstrong and Rawson, 1979). A Purkinje cell, after being penetrated by a glass microelectrode, often depolarizes to the point at which it no longer generates action potentials (Granit and Phillips, 1956; Eccles et al., 1966). The climbing fiber input is then registered as either a single EPSP or more usually as a compound EPSP composed of multiple EPSPs separated from each other by 2 ms (Eccles et al., 1966; Armstrong and Rawson, 1979). The amplitude and polarity of these compound EPSPs can be manipulated by changing the membrane potential (Eccles et al., 1966; Simpson et al., 1974), and the spike-generating mechanism can be restored by hyperpolarizing the cell (Eccles et al., 1966). The functional role of the high-frequency multiple spikes in climbing fibers is not known. Although the number of EPSPs produced by a climbing fiber burst may appear to offer a simple explanation for the number of spikelets of the complex spike (Mano, 1970; Gilbert, 1974), the number of spikelets was found to be unrelated to the number of EPSPs underlying the complex spike (Armstrong and Rawson, 1979).

By recording spontaneous activity from Purkinje cells in anes-

Received June 6, 2007; revised Aug. 23, 2007; accepted Sept. 5, 2007.

This work was supported by National Institutes of Health Grant NS-13742. We thank Dr. T. J. H. Ruigrok of Erasmus University (Rotterdam, The Netherlands) for useful discussion and Dr. C. H. Yeo of University College London (London, UK) for rekindling our interest in climbing fiber bursts.

Correspondence should be addressed to John I. Simpson, Department of Physiology and Neuroscience, 550 First Avenue, New York University School of Medicine, New York, NY 10016. E-mail: john.simpson@med.nyu.edu.

DOI:10.1523/JNEUROSCI.2559-07.2007

Copyright $\odot 2007$ Society for Neuroscience $\quad 0270-6474 / 07 / 2711263-08 \$ 15.00 / 0$ thetized rabbits, we investigated the climbing fiber bursts by determining the temporal distribution of the induced compound EPSPs according to the number of their components. Similar measurements were also made for visual modulation of climbing fiber activity in floccular Purkinje cells that responded best to visual rotation about the vertical axis (VA) (Graf et al., 1988). Our goal was to examine the possibility that the number of spikes within the climbing fiber bursts carries a signal.

\section{Materials and Methods}

Animal preparation. Six acutely prepared Dutch-belted rabbits (2.0-2.6 $\mathrm{kg}$ ) were used in this study. The experiments conformed to the Principles of Laboratory Animal Care (1996) and were approved by the Institutional Animal Care and Use Committee of New York University School of Medicine. Each animal was anesthetized with ketamine $(45 \mathrm{mg} / \mathrm{kg})$ and xylazine $(5 \mathrm{mg} / \mathrm{kg})$, and anesthesia was supplemented with additional ketamine $(10 \mathrm{mg} / \mathrm{kg})$ and xylazine $(2 \mathrm{mg} / \mathrm{kg})$ every $30-45 \mathrm{~min}$. Normal body temperature was maintained with a heating pad. During the experiment, the animal was positioned on a vertical-axis turntable with the head centered on the axis of rotation. The head was held in the "freeze" position with the nasal bone inclined at $57^{\circ}$ to the horizontal (Hughes, 1971), in which the horizontal canals were tilted $\sim 7^{\circ}$ backward and the vertical canals were essentially vertical (Simpson, 1983). The animal's eyes remained open during the course of the experiment. To allow recording of neuronal activity, an opening was made through a left superolateral portion of the occipital bone, and the dura mater overlying the cerebellum was removed.

Recording procedures. Neuronal activity was recorded with a glass pipette filled with $2-3 \mathrm{M} \mathrm{NaCl}$ solution saturated with fast green FCF (2-10 $\mathrm{M} \Omega$ ). The pipette was tilted $33^{\circ}$ forward and reached the flocculus in a parasagittal plane. The signals were amplified and bandpass filtered at $10-10,000 \mathrm{~Hz}$. Single Purkinje cell activity was extracellularly identified by the presence of complex spikes. Purkinje cells in the flocculus that 
respond best to visual whole-field rotation about either the VA or the horizontal $45^{\circ}-135^{\circ}$ axis were identified by slowly moving $(\sim 1 \%)$ a large hand-held visual pattern (Graf et al., 1988). Penetration of a Purkinje cell by the microelectrode often resulted in a period of injury discharge, after which relatively large EPSPs attributable to the climbing fiber input could be recorded (Eccles et al., 1966; Armstrong and Rawson, 1979). In the course of the depolarization of the Purkinje cell to where only waveforms with the shape of EPSPs were present, all other waveforms with a shape indicative of spike activity disappeared, leaving only EPSPs uncomplicated by spike potentials. This transition during the process of deterioration was well described by Eccles et al. (1966). As shown below in Results, the climbing fiber responses (CFRs) of an injured cell typically consisted of a substantial percentage of single-component responses, which is in contrast to Purkinje cells with an intact or partially intact mechanism for generating action potentials (Eccles et al., 1966; Armstrong and Rawson, 1979). Eliminating the regenerative components of the Purkinje cell CFR by injury has been widely used in in vivo preparations (Eccles et al., 1966; Simpson et al., 1974; Crepel et al., 1976; Armstrong and Rawson, 1979) (see also Knöpfel et al., 1990). Recording of climbing fiber-induced EPSPs could be maintained for several seconds to $>20 \mathrm{~min}$, during the course of which the amplitude of the compound EPSPs gradually diminished, with each component retaining its proportionate size. The quality and appearance of the recordings are comparable with those of previous experiments using glass pipettes filled with $3 \mathrm{M} \mathrm{KCl}$ or $2 \mathrm{M} \mathrm{K}$-citrate (Eccles et al., 1966; Crepel et al., 1976), 3 м K-acetate (Llinás and Sugimori, 1980), and $2 \mathrm{M}$ $\mathrm{NaCl}$ (Simpson et al., 1974). During the recording of some VA Purkinje cells, the animal was oscillated sinusoidally in light about the vertical axis by $\pm 10^{\circ}$ at $0.1 \mathrm{~Hz}$. Vestibular stimulation in darkness at this frequency and amplitude does not modulate the complex spike activity of floccular Purkinje cells (Simpson et al., 2002); thus, the oscillation of the anesthetized animal provided in essence whole-field visual stimulation. Neuronal activity and table position were stored on a VHS tape for off-line analyses.

Data analysis. Neuronal activity and table position were digitized at 44 $\mathrm{kHz}$ and $400 \mathrm{~Hz}$, respectively, with the use of a CED 1401 signal capture device and a Spike2 program (Cambridge Electronic Design, Cambridge, UK) on a personal computer. All the cells that were included in the analysis had records of activity during spontaneous periods lasting for at least $60 \mathrm{~s}$. The number of EPSP components in each CFR was identified as the number of positive peaks in the EPSP waveform. For simplicity of exposition, a CFR with a single EPSP is henceforth described as a compound EPSP with one component. To reiterate, the CFR of an injured Purkinje cell described in this paper is synonymous with a compound EPSP. The average frequency and composition of CFRs and the temporal relationship among compound EPSPs were determined first for each Purkinje cell and then for the sample population. Student's $t$ test, ANOVA with the post hoc Student-Newman-Keuls (SNK) test, and the Pearson's correlation coefficient were used for statistical analyses.

The modulation of the CFRs of VA Purkinje cells in response to the visual stimulus was also analyzed, provided that $60 \mathrm{~s}$ or more of relevant data were available. Excitatory and inhibitory phases of the CFR modulation of a VA cell was defined relative to counterclockwise and clockwise motions of the turntable, with the beginning of each phase corresponding to the beginning of the motion in each direction. As found previously (Graf et al., 1988), the complex spike activity of all VA Purkinje cells increased for retinal image motion in the temporal to nasal direction referenced to the eye ipsilateral to the recorded flocculus. A contrast ratio characterizing the modulation was defined as the difference in the number of CFRs between excitatory and inhibitory phases divided by the total number of CFRs during visual stimulation. By definition, the value of a contrast ratio ranges from 1 to -1 , with 0 indicating no modulation.

\section{Results}

\section{Spontaneous activity}

For our sample of 43 Purkinje cells, the average frequency of all CFRs during spontaneous activity was $1.10 \mathrm{~Hz}$ (range, 0.5-2.2 $\mathrm{Hz}$ ). A single CFR consisted of up to seven EPSPs separated from each other by 1.3-2 ms. An example of a sequence of spontaneous CFRs consisting of one to five EPSPs is shown in Figure 1. Compound EPSPs were grouped into four classes based on the number of EPSP components (one, two, three, or four or more). The average composition of compound EPSPs for all 43 cells was as follows: $17.3 \%$ for one component; $36.9 \%$ for two components; $37.3 \%$ for three components; and $8.5 \%$ for four or more components.

During spontaneous activity, the percentages of compound EPSPs with one and two components had significant negative correlations with respect to the average inter-CFR interval (Fig. $2 A, B)$. Conversely, the percentages of compound EPSPs with three and four or more components had significant positive correlations with respect to the average inter-CFR interval (Fig. $2 C, D)$. Thus, compared with cells with high spontaneous CFR rates, cells with lower spontaneous CFR rates tended to have fewer compound EPSPs with one or two components and more compound EPSPs with three or more components.

We asked whether the number of components in each compound EPSP was related to the preceding or succeeding interCFR interval and could, therefore, account for the correlations between the percentages of different compound EPSP components and the spontaneous CFR rates. We found that the number of components in a compound EPSP was not related to the average latency to the succeeding CFR (Fig. $3 A$ ) (ANOVA, $F_{(3,154)}=$ 1.51). Therefore, an occurrence of a CFR, regardless of the number of its EPSP components, did not predict the average timing of the next CFR. This apparent memoryless characteristic is in agreement with the finding of Armstrong and Rawson (1979). In contrast, the number of components in a compound EPSP was related to the latency after the preceding CFR (ANOVA, $F_{(3,154)}=$ 30.29; $p<0.001$ ). The average latency increased monotonically from $0.62 \mathrm{~s}$ for one-component EPSPs to $1.69 \mathrm{~s}$ for compound EPSPs with four or more components (Fig. $3 B$ ), and all pairwise 

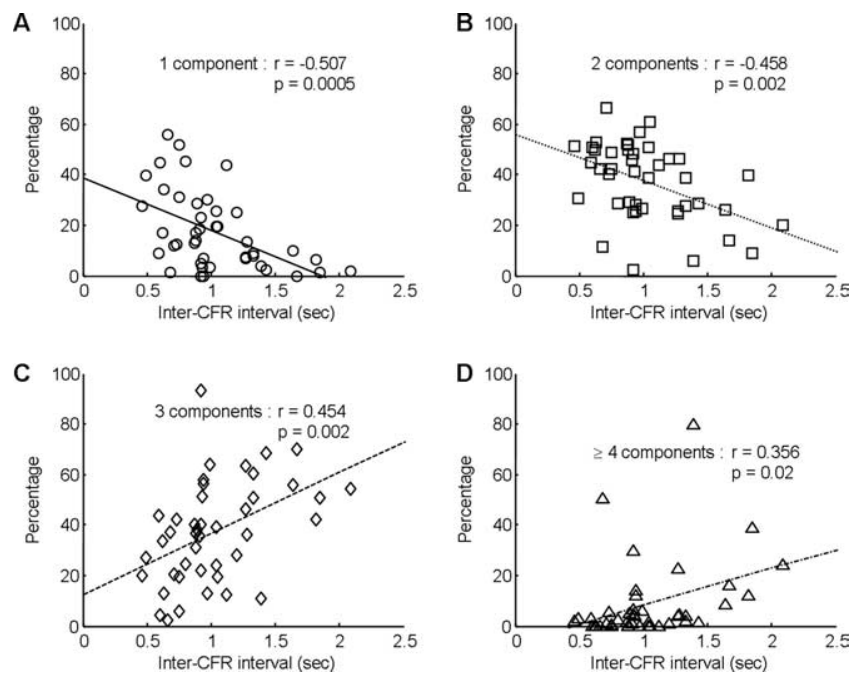

Figure 2. Percentages of compound EPSPs with different numbers of components plotted with respect to mean inter-CFR interval (data from 43 Purkinje cells). $A$, One-component EPSPS. B, Two-component compound EPSPS. C, Three-component compound EPSPs. D, Compound EPSPs with four or more components. Pearson's correlation coefficient $(r)$ and significance level ( $p$ ) are displayed for each panel.

differences were significant $(p<0.05$, one component vs two component; $p<0.01$, all other pairs, SNK test). In other words, the larger the number of components in a compound EPSP, the longer was its average latency after the preceding CFR. Thus, the correlations between the percentages of compound EPSPs with different numbers of components and the spontaneous CFR rates (Fig. 2) are accounted for by the relationship between the number of components in a compound EPSP and the preceding interCFR interval.

To study the latency distribution of compound EPSPs, the available records for each Purkinje cell during spontaneous activity, varying from 60 to $400 \mathrm{~s}$ in duration, were combined. This procedure is justified because the variability in the durations was a random variable beyond the control of the experimenters, and the ratio between the longest and the shortest durations was reasonably small. The procedure is also justified because the values of parameters, including the average inter-CFR interval, the average percentages of compound EPSPs with different numbers of components, and the average latencies before and after compound EPSPs with different numbers of components, were essentially the same as those derived with an equal weight given to each Purkinje cell regardless of the length of recording. The histogram distribution of the inter-CFR intervals was positively skewed, tapering to longer intervals (Fig. $4 A$ ). The average interval was $0.94 \mathrm{~s}$ (i.e., $1.06 \mathrm{~Hz}$ average frequency). Within this distribution, the composition of compound EPSPs with different numbers of components changed with the latency after the preceding CFR (Fig. $4 B$ ). The percentage of one-component EPSPs (solid line with circles) was $\sim 40 \%$ for the first $0.2 \mathrm{~s}$ bin immediately after the preceding compound EPSP and declined to $\sim 5 \%$ within $3 \mathrm{~s}$. The percentage of two-component compound EPSPs (dotted line with squares) also declined, but from $\sim 45$ to $\sim 20 \%$. Conversely, the percentage of three-component compound EPSPs (dashed line with diamonds) was $\sim 15 \%$ for the first $0.2 \mathrm{~s}$ bin immediately after the preceding compound EPSP and increased to 55\% over time. Similarly, the percentage of compound EPSPs with four or more components (dot-dashed line with triangles) increased from essentially 0 to $\sim 20 \%$. Accordingly, the occurrences of each class of compound EPSP with a different number of components
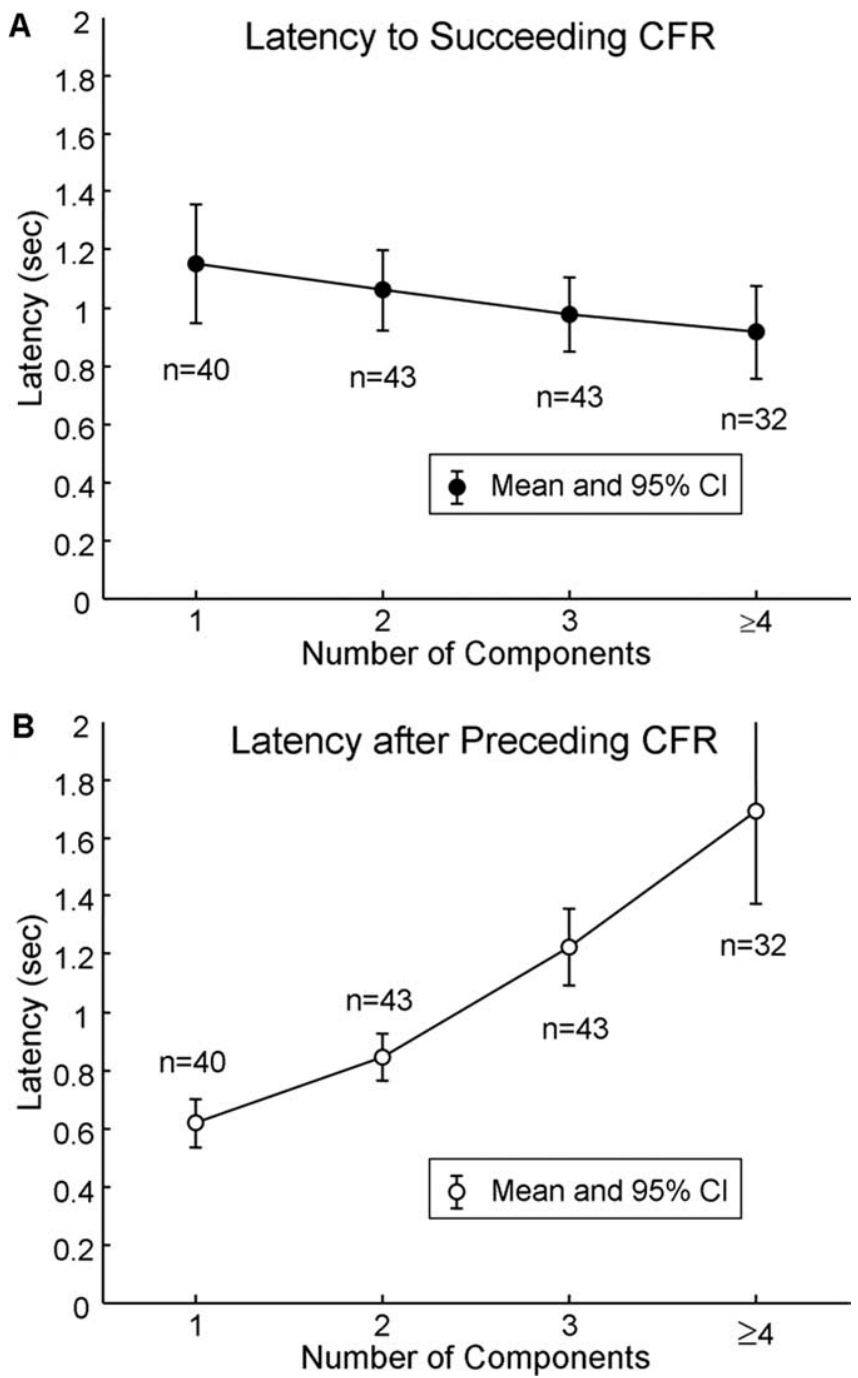

Figure 3. Relationship between the number of components in the compound EPSPs and latencies. $\boldsymbol{A}$, Relationship between the number of EPSP components and the latency to the succeeding CFR. The means and 95\% confidence intervals ( $\mathrm{Cl}$ ) are plotted. $\boldsymbol{B}$, Relationship between the number of EPSP components and the latency after the preceding CFR. $A, B$, The data were taken from 43 Purkinje cells, but some cells lacked one-component or four- or more component compound EPSPs during the recording period, as indicated by $n<43$.

had a distinctive distribution over time (Fig. 4C), with the mean increasing with the number of components.

In contrast, the percentage of CFRs that succeeded each class of compound EPSP with a particular number of components (i.e., one, two, three, or four or more) did not change with time (Fig. 4D). That is, no class of compound EPSP was predisposed to be succeeded at any preferred latency by the next CFR. That the number of EPSP components in a CFR is not related to the latency to the succeeding CFR is consistent with the notion of memorylessness as described above.

\section{Activity during visual stimulation}

In 19 VA Purkinje cells, the effect of whole-field visual stimulation was investigated by sinusoidally oscillating the animal by $\pm 10^{\circ}$ about the vertical axis at $0.1 \mathrm{~Hz}$. When both the excitatory and inhibitory phases of the response to visual stimulation were considered together, the overall CFR frequency increased to 1.17 from $1.07 \mathrm{~Hz}$ for spontaneous activity (paired $t$ test, $p<0.01 ; n=19$ ).

The average distribution of CFRs during visual stimulation 

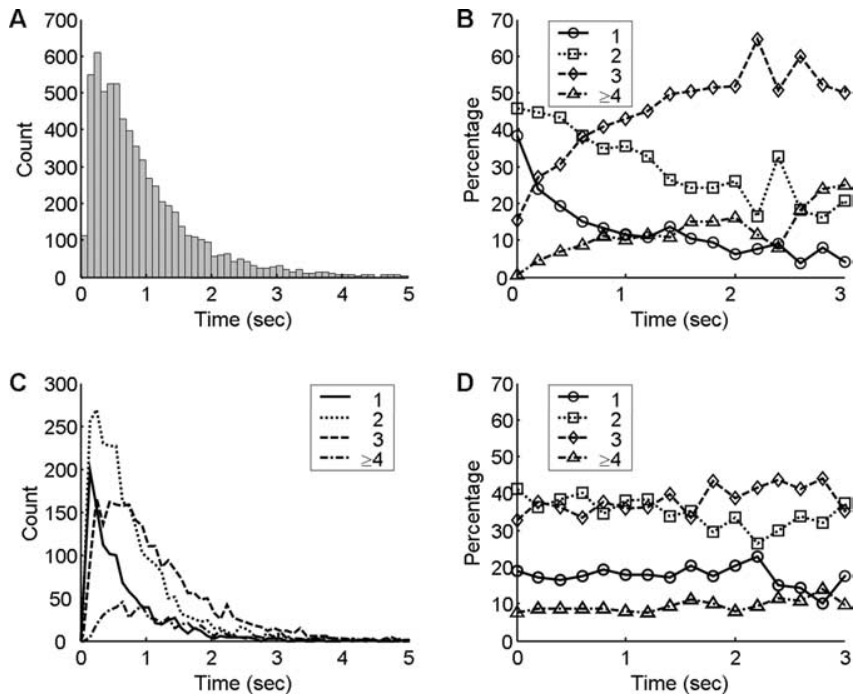

Figure 4. Inter-CFR dynamics. $A$, Histogram of inter-CFR interval distribution (bin width, $0.1 \mathrm{~s}$ ). $\boldsymbol{B}$, Percentages of compound EPSPs with different numbers of components (indicated in the inset) as a function of latency after the preceding (FR (bin width, $0.2 \mathrm{~s}$ ). C, Breakdown of $\boldsymbol{A}$ for the different number of components in the compound EPSP. D, Percentages of CFRs as a function of latency after compound EPSPs with different numbers of components (indicated in the inset; bin width, $0.2 \mathrm{~s}$ ). $\boldsymbol{B}-\boldsymbol{D}$, One-component EPSPs, solid line with or without circles; two-component compound EPSPs, dotted line with or without squares; three-component compound EPSPs, dashed line with or without diamonds; compound EPSPs with four or more components, dot-dashed line with or without triangles. Note that, for $\boldsymbol{B}$ and $\boldsymbol{D}$, the total percentage adds up to 100 for each $0.2 \mathrm{~s}$ bin width.

was characterized as the probability of occurrence within each $0.2 \mathrm{~s}$ bin (50 bins per cycle). Because the peak velocity of the sinusoidal stimulus $(6.3 \%)$ was beyond the optimal response range of VA Purkinje cells (Graf et al., 1988), the excitatory phase of the response (Fig. 5A, right half) consisted of an initial transient rise, a fall to a near average level (dotted horizontal line), and a second rise as the stimulus velocity fell before reversing direction. The inhibitory phase also consisted of three analogous subphases (Fig. 5A, left half): namely, a decrease in response, a return to a near average level, and a second decrease. The contrast ratio (see Materials and Methods) composed of both the excitatory and inhibitory phases of all CFRs during visual stimulation was 0.59. All 19 cells responded with one-, two-, and threecomponent compound EPSPs, but only 15 cells responded with compound EPSPs with four or more components during the stimulus. The contrast ratios for compound EPSPs with different numbers of components were not equal (ANOVA, $F_{(3,68)}=$ 14.94; $p<0.001$ ) and monotonically increased from 0.33 for one-component EPSPs to 0.90 for compound EPSPs with four or more components, indicating a deeper modulation for compound EPSPs with a larger number of components (Fig. 5B-E). Thus, climbing fibers can, in addition to modulation of their low interburst frequency, transmit signals in the form of the number of spikes within each high-frequency burst.

Accordingly, the composition of compound EPSPs was different during excitatory and inhibitory phases (two-way ANOVA interaction, $\left.F_{(3,144)}=6.32 ; p<0.001\right)$. The percentage of onecomponent EPSPs (Fig. 6, leftmost group) during the inhibitory phase $(26.1 \%$, black frame) was significantly larger than that for both spontaneous activity [16.2\% (dashed frame); paired $t$ test, $p<0.05 ; n=19]$ and for the excitatory phase [10.5\% (white frame); paired $t$ test, $p<0.001 ; n=19$ ]. In contrast, the percentage of three-component compound EPSPs (Fig. 6, third group from left) during the inhibitory phase $(27.5 \%)$ was smaller than that for both spontaneous activity (37.1\%; paired $t$ test, $p<$ $0.001 ; n=19)$ and for the excitatory phase (39.5\%; paired $t$ test, $p<0.001 ; n=19)$. The overall percentage of compound EPSPs with four or more components (rightmost group) during visual stimulation increased significantly to $8.9 \%$ (gray frame) from $5.8 \%$ for spontaneous activity (paired $t$ test, $p<0.05 ; n=19$ ). Just as for the three-component compound EPSPs, the percentage of compound EPSPs with four or more components during the inhibitory phase $(3.6 \%)$ was smaller than that during the excitatory phase $(9.7 \%$; paired $t$ test, $p<0.05 ; n=19)$, which in turn was larger than that for spontaneous activity (5.8\%; paired $t$ test, $p<0.05 ; n=19)$. Thus, the change in the composition of compound EPSPs between excitatory and inhibitory phases pivoted about the two-component compound EPSPs, whose percentage remained statistically unchanged from the spontaneous level for both the excitatory and inhibitory phases.

We examined whether visual stimulation altered the relationship found for spontaneous activity between the number of EPSP components and the latency after the preceding CFR. With both the excitatory and inhibitory phases of the responses combined, the number of components in the compound EPSPs in the 19VA Purkinje cells depended on the latency after the preceding CFR (ANOVA, $\left.F_{(3,68)}=10.92 ; p<0.001\right)$. Like the relationship found for spontaneous activity (ANOVA, $F_{(3,64)}=27.08$; $p<0.001$ ), the average latency was larger for compound EPSPs with a larger number of components (Fig. 7). However, the latency relationship was not as strong during visual stimulation (filled triangles) as during spontaneous activity (open circles). Although all pairwise differences were significant for spontaneous activity, for activity during visual stimulation, the latency difference between one- and two-component compound EPSPs and that between three- and four- or more component compound EPSPs were not significant (SNK test). Moreover, the average latency after the preceding CFR for compound EPSPs with four or more components was significantly shorter during visual stimulation than during spontaneous activity (one-tailed $t$ test, $p<0.05$; $n=15$, 12). Nevertheless, because there was only a small increase in the overall CFR frequency during visual stimulation $(1.17 \mathrm{~Hz})$ from that during spontaneous activity $(1.07 \mathrm{~Hz})$, the difference in the composition of compound EPSPs between excitatory and inhibitory phases of visual stimulus was not great enough to override the latency relationship found for spontaneous activity.

\section{Stochastic model}

A stochastic model was constructed to test whether the distribution of inter-CFR intervals during spontaneous activity as well as the relationship between the number of components in a compound EPSP and the latency after the preceding CFR could be simulated. After the initial few hundred milliseconds indicative of a refractoriness, the distribution of interburst intervals decayed with a decreasing rate (Fig. $4 A$ ). Thus, by letting the process of recovery from refractoriness form an envelope over the decay process, the probability density distribution of interburst intervals, $P$, can be represented as follows:

$$
P=\frac{\tau_{1}+\tau_{2}}{\tau_{2}^{2}}\left(1-e^{-t / \tau_{1}}\right) e^{-t / \tau_{2}},
$$

where $t$ is the latency of a CFR after the preceding CFR, $\tau_{1}$ is the time constant that controls the recovery from refractoriness, and $\tau_{2}$ is the time constant of a Poisson process that is independent of the preceding CFR and acts as a trigger for the next discharge 

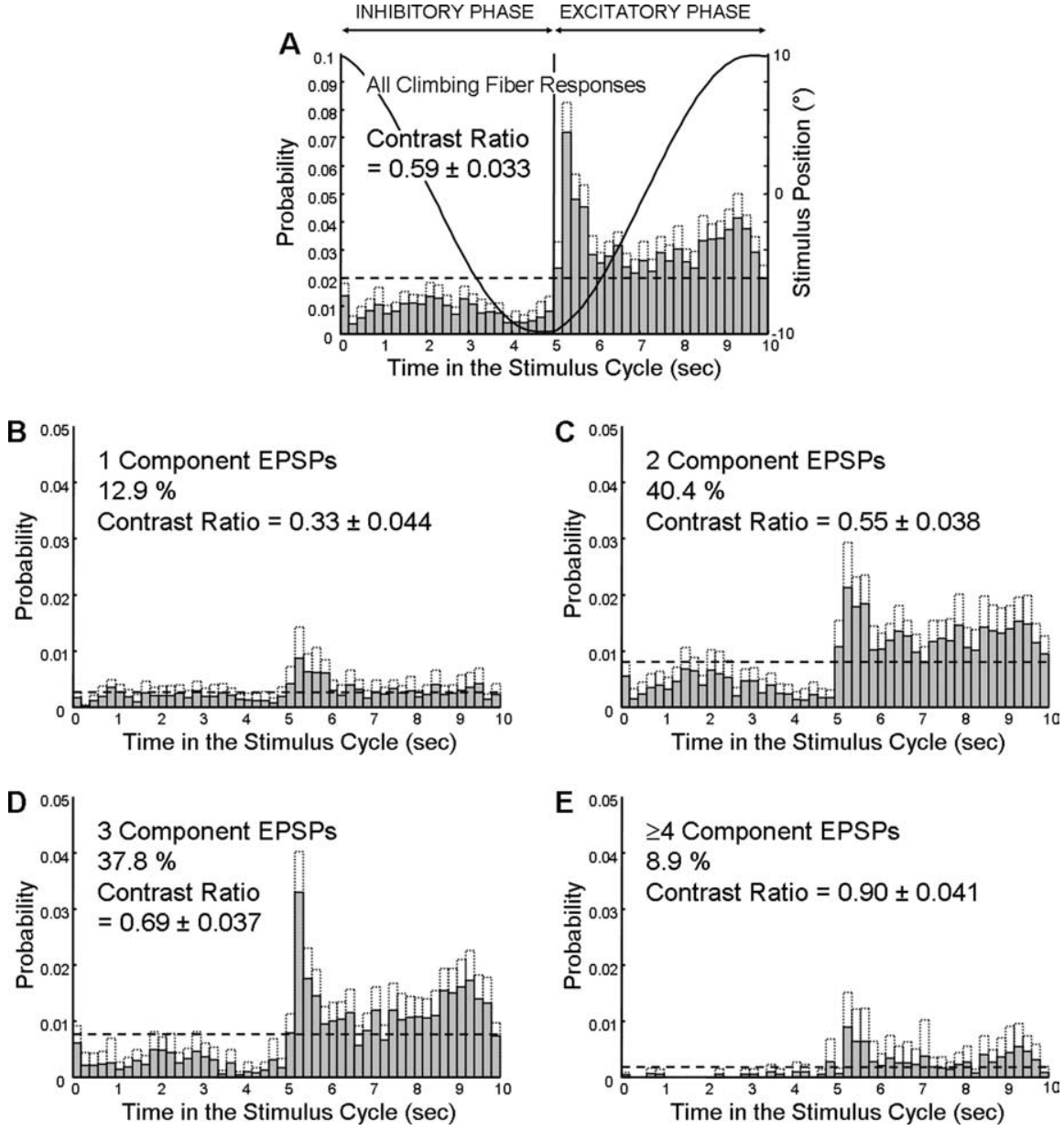

Figure 5. Probability histograms of average distribution of responses in 19 VA Purkinje cells during the stimulus cycle. $\boldsymbol{A}$, All CFRs. $\boldsymbol{B}-\boldsymbol{E}$, One-component and multicomponent compound EPSPS. The dashed horizontal line across each panel indicates the expected probability of events per bin $(0.2 \mathrm{~s})$ if there were no modulation. The dotted line over each bar indicates the upper limit of the $95 \%$ confidence interval. In $\boldsymbol{A}$, the table position is overlaid to indicate the relationship between the stimulus and the response.

from the inferior olive. Consequently, consecutive interburst intervals are independent of each other. This formulation allows for an explicit constraint on $\tau_{1}$. The value of $\tau_{1}$ was set to $0.1 \mathrm{~s}$, compatible with the previously reported duration of refractoriness (Llinás and Yarom, 1981ab; Bloedel and Ebner, 1984). With the value of $\tau_{1}$ set, the value of $\tau_{2}$ was determined to be $0.85 \mathrm{~s}$ so that the average interburst interval was equal to the experimentally measured value of $0.94 \mathrm{~s}$ (Fig. $8 \mathrm{~A}$ ).

As illustrated in Figure $4 B$, the percentage of compound EPSPs with a particular number of components changed with time after the preceding CFR. Exponential fits of these changes yielded time constants similar to $\tau_{2}$. Thus, the composition of compound EPSPs corresponding to the combination of the individual percentages, $q(1)$ through $q(\geq 4)$, can be expressed by the following:

$$
\begin{gathered}
q(1)=0.35 \times e^{-t / \tau_{2}}+0.05 \\
q(2)=0.25 \times e^{-t / \tau_{2}}+0.2 \\
q(3)=0.4 \times e^{-t / \tau_{2}}+0.55 \\
q(\geq 4)=-0.2 \times e^{-t / \tau_{2}}+0.2,
\end{gathered}
$$

as shown in (Fig. $8 \mathrm{~B}$ ). The positive coefficients of the exponential terms for $q(1)$ and $q(2)$ and negative coefficients for $q(3)$ and $q(\geq 4)$ characterize, respectively, the rise and fall of these functions with respect to time. The product of $P$ and $q(n)$, where $n$ is the number of EPSP components, determines the simulated probability density distribution of each compound EPSPs with $n$ components (Fig. $8 C$ ). The average latencies of the model EPSPs monotoni-

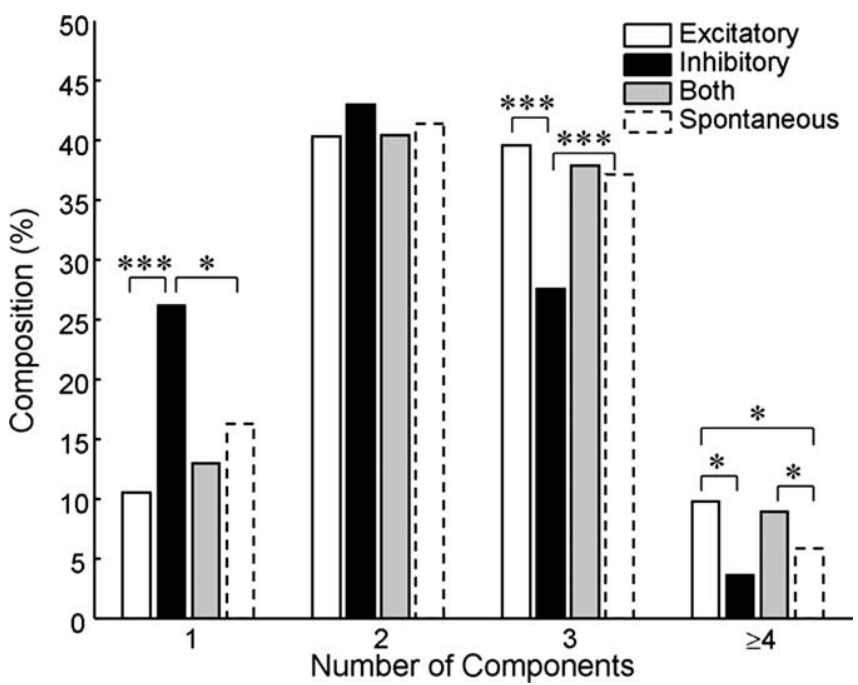

Figure 6. Percentages of the number of components in the compound EPSPs in 19 VA Purkinje cells during the excitatory phase (white frame), inhibitory phase (black), and entire visual stimulation period (gray). The corresponding percentages for spontaneous activity are also shown (dashed frame). ${ }^{*} p<0.05 ;{ }^{* * *} p<0.001$. cally increase with the number of EPSP components in a manner comparable with the experimental data (Fig. $8 D$ ).

\section{Discussion}

We found that, for spontaneous climbing fiber activity, the larger the number of EPSP components in a CFR of a Purkinje cell, the more likely it is that its latency after the preceding CFR is increased. That is, the number of climbing fiber intraburst spikes probabilistically depends on the latency after the preceding climbing fiber discharge. In addition, we found that, during visual stimulation, climbing fibers can, in addition to modulation of their low interburst frequency, transmit signals in the form of the number of spikes within each high-frequency intraburst.

\section{Mechanism of burst generation}

The precise mechanism that determines the number of intraburst spikes or the length of the interburst interval is not known. The original hypothesis that the climbing fiber bursts originate from positive feedback to the inferior olivary neurons through collaterals (Eccles et al., 1966) has been ruled out anatomically (Ruigrok et al., 1990; De Zeeuw et al., 1996). Instead, an axonal burst is generated through an extended somatic afterdepolariza- 


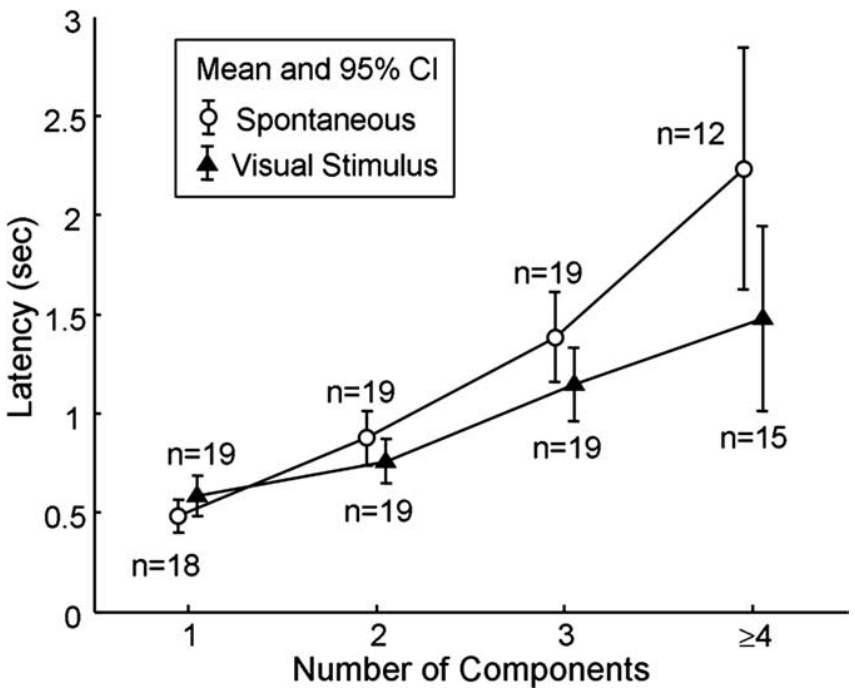

Figure 7. Relationship for VA Purkinje cells between the number of components in a compound EPSP and the latency after the preceding (FR during spontaneous activity (open circles) and during visual stimulation (filled triangles). The means and $95 \%$ confidence intervals (Cl) are plotted. The data were taken from 19 cells, but some of them lacked one-component or four- or more component compound EPSPs during the recording period, as indicated by $n<19$.
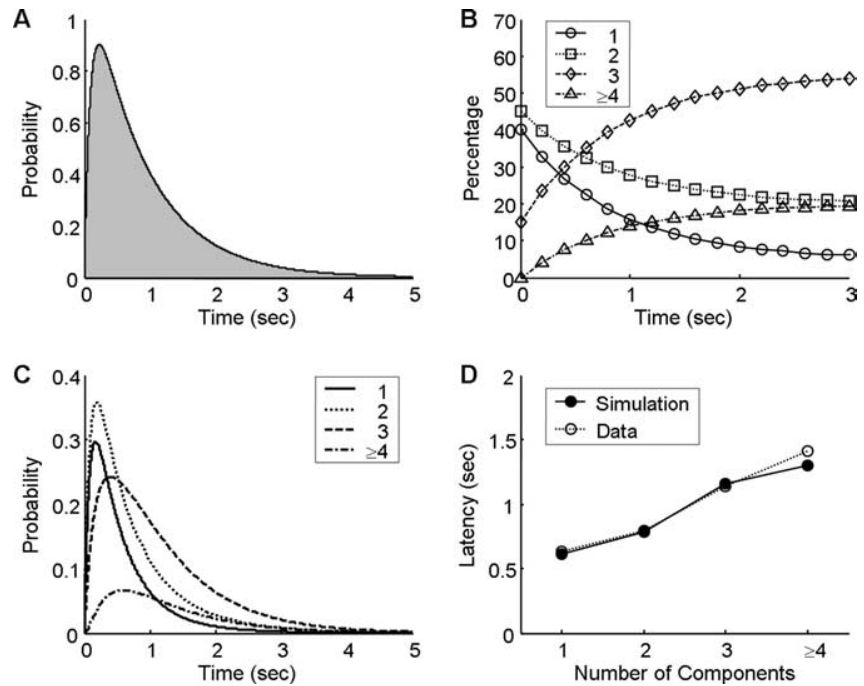

Figure 8. Stochastic model of CFR dynamics. $\boldsymbol{A}$, The probability density distribution of CFR intervals modeled as a double-exponential function. $\boldsymbol{B}$, Percentages of compound EPSPs with different numbers of components (indicated in the inset) modeled as exponential functions of latency after the preceding (FR. Note that the total percentage adds up to 100 at any given time. C, The probability density distribution of model compound EPSPs with different numbers of components. B, C, One-component EPSPs, solid line with or without circles; two-component compound EPSPs, dotted line with or without squares; three-component compound EPSPS, dashed line with or without diamonds; compound EPSPs with four or more components, dotdashed line with or without triangles. $\boldsymbol{D}$, Relationship between the number of components in a compound EPSP and the average latency after the preceding CFR. Solid line with filled circles, Model simulation; dotted line with open circles, experimental data.

tion that often accompanies the initial action potential in an olivary neuron (Crill and Kennedy, 1967; Crill, 1970; Llinás and Yarom, 1981a; Ruigrok and Voogd, 1995). The duration of this afterdepolarization is determined by an interplay between dendritic $\mathrm{Ca}^{2+}$ and $\mathrm{K}^{+}$conductances (Llinás and Yarom, 1981b), but the precise nature of the interplay has not been determined.

Based on the finding that olivary neurons were as likely to produce multiple spikes with a just-threshold electrical stimulus as with a stronger one (Eccles et al., 1966; Crill, 1970), it was hypothesized that, whereas the summed synaptic inputs evoke somatic action potentials and determine the interburst intervals, the number of intraburst spikes is determined by some intrinsic property of the neuron that controls the duration of the afterdepolarization (Crill, 1970). However, we found that, during whole-field visual stimulation, a natural stimulus, both the frequency of firing and the number of intraburst spikes were modulated. Therefore, it is likely that the duration of the afterdepolarization is also influenced by the combined synaptic inputs to the olivary neuron. It has been shown that the triggering of an action potential in an olivary neuron and the accompanying afterdepolarization are both influenced by the resting potential of the cell (Llinás and Yarom, 1981a; Ruigrok and Voogd, 1995). Thus, in addition to the excitatory inputs to the inferior olive from various sources, the GABAergic input from the deep cerebellar nuclei that forms a feedback loop via the cerebellar cortex (Andersson et al., 1988; Ruigrok and Voogd, 1995) (for review, see Bengtsson and Hesslow, 2006) may also be an important determinant that regulates the compositions of the burst of olivary neurons.

\section{Stochastic model}

A model using only a small set of parameters successfully simulated the distribution of inter-CFR intervals as well as the relationship between the number of components in a compound EPSP and the latency after the preceding CFR during spontaneous activity. What can be revealed by the model? First, because of the assumptions made in the model, the similarity of the model outcome to the data implies that the number of EPSP components in a CFR does not depend on the number of EPSP components in the preceding CFR but, as found, on the latency after it. Second, involvement of relatively few parameters in the model implies that the complexity of the mechanism that controls climbing fiber bursts may be explained in terms of a small number of subprocesses. For instance, the agreement between the time constant for the Poisson process, $\tau_{2}$, and the time constant for the change in the EPSP composition may indicate that the two processes share the same mechanism, although a similarity between these parameters for individual cells could not be dependably established for our data. Third, the model implies that the EPSP composition $[q(1), q(2), q(3)$, and $q(\geq 4)]$ is not predisposed to an abrupt change. This postulate is consistent with the finding that the relationship between the number of EPSP components and the latency after the preceding CFR is maintained during visual stimulation, albeit not as strongly as during spontaneous activity. Consequently, provided that the percentages of the various number of climbing fiber intraburst spikes are weighted with an additional element so as to increase the percentages of bursts with a larger number of spikes during excitation, the model may be adapted to simulate responses during visual stimulation.

\section{Functions of climbing fiber bursts}

Because a single action potential in a climbing fiber can evoke a variable number of spikelets in a Purkinje cell complex spike recorded near the soma (Eccles et al., 1966), the number of EPSP components underlying a Purkinje cell complex spike is unrelated to the number of spikelets in the complex spike, as also shown directly by Armstrong and Rawson (1979). Therefore, the functional significance of the number of components in compound EPSPs is not immediately evident. Similarly, a relationship between the propagation of Purkinje cell complex spike ac- 
tivity as axonal spikes (Ito and Simpson, 1971; Campbell and Hesslow, 1986; Khaliq and Raman, 2005; Monsivais et al., 2005) and the number of EPSP components underlying the complex spike is unknown. Potential roles of climbing fiber bursts may be multifold because influences on cerebellar interneurons by climbing fibers include direct synaptic excitation as well as longterm potentiation (Jörntell and Ekerot, 2003). However, because there are fewer synaptic contacts by collaterals of climbing fibers in the deep cerebellar nuclei and on cerebellar interneurons than those made by a climbing fiber onto a Purkinje cell (Shinoda et al., 2000), Purkinje cells are likely to be the cells affected most directly by the number of climbing fiber intraburst spikes.

One effect that follows climbing fiber activation in a Purkinje cell is a brief inactivation of simple spikes lasting $\sim 15-30 \mathrm{~ms}$ (Granit and Phillips, 1956; Thach, 1967; Bell and Grimm, 1969). This inactivation, or pause, may be caused both by the direct action of the climbing fiber on the Purkinje cell and by inhibition of the Purkinje cell via activation of the neighboring interneurons through the climbing fiber collaterals (Murphy and Sabah, 1970; Bloedel and Roberts, 1971; Colin et al., 1980). After the pause, the spontaneous simple spike activity of a Purkinje cell may be facilitated, unchanged, or reduced (McDevitt et al., 1982; Sato et al., 1992). The period of inactivation varies from complex spike to complex spike (McDevitt et al., 1982), but, when Purkinje cells are statistically categorized into pause-facilitation, pure-pause, and pause-reduction types, there is a trend associating these cell types with high, medium, and low average frequency of complex spikes, respectively (Sato et al., 1992). We found a correlation between the average rate of the CFR and the number of EPSP components in a CFR, but whether the number of climbing fiber intraburst spikes plays a role in short-term changes in simple spike activity is not known. Such a function, if it exists, may also complement the role of climbing fibers in regulating the level of Purkinje cell simple spike firing (Colin et al., 1980; Cerminara and Rawson, 2004).

Another effect of climbing fiber activation on a Purkinje cell is induction of long-term depression (LTD). The magnitude of the LTD of parallel fiber-Purkinje cell synapses has been shown to depend on the stimulation frequency (Ekerot and Kano, 1989; Karachot et al., 1994). Conjunctive stimulation of climbing fiber and parallel fiber pathways in vivo at $4 \mathrm{~Hz}$ is more effective in inducing LTD than is stimulation at 1 or $2 \mathrm{~Hz}$ (Ekerot and Kano, 1989). This observation suggests a greater efficacy with highfrequency stimulation, although continuous stimulation of the climbing fiber pathway at above $4 \mathrm{~Hz}$ induces a prolonged depression of the Purkinje cell responsiveness in a nonselective manner (Ito et al., 1982). It has been shown in vitro that stimulation of climbing fibers with trains of three to five electrical pulses with short intervals $(10-50 \mathrm{~ms})$ repeated every 5-40 s is effective in inducing parallel fiber LTD (Karachot et al., 1994; Schreurs et al., 1996). The effects of a more physiologically realistic stimulus, i.e., two to five pulses $\sim 2 \mathrm{~ms}$ apart, with various rates of repetition should be investigated (Jirenhed et al., 2007). The more recently discovered LTD of climbing fiber-Purkinje cell synapses that is coupled with a change in the complex spike waveform (Hansel and Linden, 2000) is another phenomenon that may be affected by climbing fiber bursts.

Although there is no consensus on the overall function of climbing fibers (for review, see Simpson et al., 1996), there is agreement that they are involved in controlling the background Purkinje cell firing. In addition, many investigators agree that climbing fibers are critical for cerebellar learning. However, the previously well documented burst property of climbing fiber ac- tivity (Eccles et al., 1966; Crepel et al., 1976; Armstrong and Rawson, 1979) has been largely overlooked in recent investigations. The present finding that the number of spikes within the climbing fiber bursts carries a signal may have an important consequence for understanding the function of the climbing fiber input to the cerebellum.

\section{References}

Andersson G, Garwicz M, Hesslow G (1988) Evidence for a GABAmediated cerebellar inhibition of the inferior olive in the cat. Exp Brain Res 72:450-456.

Armstrong DM, Rawson JA (1979) Activity patterns of cerebellar cortical neurons and climbing fibre afferents in the awake cat. J Physiol (Lond) 289:425-448.

Bell CC, Grimm RJ (1969) Discharge properties of Purkinje cells recorded on single and double microelectrodes. J Neurophysiol 32:1044-1055.

Bengtsson F, Hesslow G (2006) Cerebellar control of the inferior olive. Cerebellum 5:7-14.

Bloedel JR, Ebner TJ (1984) Rhythmic discharge of climbing fibre afferents in response to natural peripheral stimuli in the cat. J Physiol (Lond) 352:129-146.

Bloedel JR, Roberts WJ (1971) Action of climbing fibers in cerebellar cortex of the cat. J Neurophysiol 34:17-31.

Campbell NC, Hesslow G (1986) The secondary spikes of climbing fibre responses recorded from Purkinje cell axons in cat cerebellum. J Physiol (Lond) 377:225-235.

Cerminara NL, Rawson JA (2004) Evidence that climbing fibers control an intrinsic spike generator in cerebellar Purkinje cells. J Neurosci 24:4510-4517.

Colin F, Manil J, Desclin JC (1980) The olivocerebellar system. I. Delayed and slow inhibitory effects: an overlooked salient feature of cerebellar climbing fibers. Brain Res 187:3-27.

Crepel F, Mariani J, Delhaye-Bouchaud N (1976) Evidence for a multiple innervation of Purkinje cells by climbing fibers in the immature rat cerebellum. J Neurobiol 7:567-578.

Crill WE (1970) Unitary multiple-spiked responses in cat inferior olive nucleus. J Neurophysiol 33:199-209.

Crill WE, Kennedy T (1967) Inferior olive of the cat: intracellular recording. Science 157:716-718.

De Zeeuw CI, Lang EJ, Sugihara I, Ruigrok TJ, Eisenman LM, Mugnaini E, Llinas R (1996) Morphological correlates of bilateral synchrony in the rat cerebellar cortex. J Neurosci 16:3412-3426.

Eccles JC, Llinás R, Sasaki K (1966) The excitatory synaptic action of climbing fibres on the Purkinje cells of the cerebellum. J Physiol (Lond) 182:268-296.

Ekerot CF, Kano M (1989) Stimulation parameters influencing climbing fibre induced long-term depression of parallel fibre synapses. Neurosci Res 6:264-268.

Gilbert PF (1974) A theory of memory that explains the function and structure of the cerebellum. Brain Res 70:1-18.

Graf W, Simpson JI, Leonard CS (1988) Spatial organization of visual messages of the rabbit's cerebellar flocculus. II. Complex and simple spike responses of Purkinje cells. J Neurophysiol 60:2091-2121.

Granit R, Phillips CG (1956) Excitatory and inhibitory processes acting upon individual Purkinje cells of the cerebellum in cats. J Physiol (Lond) 133:520-547.

Hansel C, Linden DJ (2000) Long-term depression of the cerebellar climbing fiber-Purkinje neuron synapse. Neuron 26:473-482.

Hughes A (1971) Topographical relationships between the anatomy and physiology of the rabbit visual system. Doc Ophthalmol 30:30-159.

Ito M, Simpson JI (1971) Discharges in Purkinje cell axons during climbing fiber activation. Brain Res 31:215-219.

Ito M, Sakurai M, Tongroach P (1982) Climbing fibre induced depression of both mossy fibre responsiveness and glutamate sensitivity of cerebellar Purkinje cells. J Physiol (Lond) 324:113-134.

Jirenhed DA, Bengtsson F, Hesslow G (2007) Acquisition, extinction, and reacquisition of a cerebellar cortical memory trace. J Neurosci 27:2493-2502.

Jörntell H, Ekerot CF (2003) Receptive field plasticity profoundly alters the cutaneous parallel fiber synaptic input to cerebellar interneurons in vivo. J Neurosci 23:9620-9631.

Karachot L, Kado RT, Ito M (1994) Stimulus parameters for induction of 
long-term depression in in vitro rat Purkinje cells. Neurosci Res 21:161-168.

Khaliq ZM, Raman IM (2005) Axonal propagation of simple and complex spikes in cerebellar Purkinje neurons. J Neurosci 25:454-463.

Knöpfel T, Audinat E, Gahwiler BH (1990) Climbing fibre responses in olivo-cerebellar slice cultures. I. Microelectrode recordings from Purkinje cells. Eur J Neurosci 2:726-732.

Llinás R, Sugimori M (1980) Electrophysiological properties of in vitro Purkinje cell somata in mammalian cerebellar slices. J Physiol (Lond) 305:171-195.

Llinás R, Yarom Y (1981a) Electrophysiology of mammalian inferior oliveary meirpms in vitro. Different types of voltage-dependent ionic conductances J Physiol (Lond) 315:549-567.

Llinás R, Yarom Y (1981b) Properties and distribution of ionic conductances generating electroresponsiveness of mammalian inferior olivary neurons in vitro. J Physiol (Lond) 315:569-584.

Mano N (1970) Changes of simple and complex spike activity of cerebellar Purkinje cells with sleep and waking. Science 170:1325-1327.

McDevitt CJ, Ebner TJ, Bloedel JR (1982) The changes in Purkinje cell simple spike activity following spontaneous climbing fiber inputs. Brain Res 237:484-491.

Monsivais P, Clark BA, Roth A, Hausser M (2005) Determinants of action potential propagation in cerebellar Purkinje cell axons. J Neurosci 25:464-472.

Murphy JT, Sabah NH (1970) The inhibitory effect of climbing fiber activation on cerebellar Purkinje cells. Brain Res 19:486-490.
Ruigrok TJ, Voogd J (1995) Cerebellar influence on olivary excitability in the cat. Eur J Neurosci 7:679-693.

Ruigrok TJ, de Zeeuw CI, van der Burg J, Voogd J (1990) Intracellular labeling of neurons in the medial accessory olive of the cat. I. Physiology and light microscopy. J Comp Neurol 300:462-477.

Sato Y, Miura A, Fushiki H, Kawasaki T (1992) Short-term modulation of cerebellar Purkinje cell activity after spontaneous climbing fiber input. J Neurophysiol 68:2051-2062.

Schreurs BG, Oh MM, Alkon DL (1996) Pairing-specific long-term depression of Purkinje cell excitatory postsynaptic potentials results from a classical conditioning procedure in the rabbit cerebellar slice. J Neurophysiol 75:1051-1060.

Shinoda Y, Sugihara I, Wu HS, Sugiuchi Y (2000) The entire trajectory of single climbing and mossy fibers in the cerebellar nuclei and cortex. Prog Brain Res 124:173-186.

Simpson JI (1983) Transformations of coordinates intrinsic to the vestibulo-ocular reflex. Soc Neurosci Abstr 9:95.4.

Simpson JI, Precht W, Llinás R (1974) Sensory separation in climbing and mossy fiber inputs to cat vestibulocerebellum. Pflügers Arch 351:183-193.

Simpson JI, Wylie DR, De Zeeuw CI (1996) On climbing fiber signals and their consequence(s). Behav Brain Sci 19:384-398.

Simpson JI, Belton T, Suh M, Winkelman B (2002) Complex spike activity in the flocculus signals more than the eye can see. Ann NY Acad Sci 978:232-236.

Thach WT (1967) Somatosensory receptive fields of single units in cat cerebellar cortex. J Neurophysiol 30:675-696. 